\title{
Foam layered apparatus with foam stabilization
}

\author{
Liaposhchenko A. $^{1}$, Khukhryanskiy O. ${ }^{2}$, Moiseev V. ${ }^{3}$, Manoilo E. ${ }^{4}$ \\ 1-2. Sumy State University, Ukraine, Sumy, 2 Rymskogo-Korsakova St., 40007, lyaposchenko@ukr.net \\ 3-4. KhTPE, NTU “KhPI”, Ukraine, Kharkiv, 2 Kyrpychova str., 61002, E-mail: bublikova1@gmail.com
}

\begin{abstract}
In work were studied the expanded models of foaming apparatuses with gratings, made of tubes of different diameters. The questions of intensification of foam devices with the use of coarsegrating lattices are considered. The possibility of deep cleaning and practically complete cleaning of gases from ammonia and fluoride compounds with their separate absorption is noted.
\end{abstract}

Keywords - industrial gas emissions; cleaning process; hydrodynamics, mass transfer; foam apparatus; foam layer, research of purification processes; stabilization of foam layer; intensive apparatus

\section{Introduction}

In order to reduce energy consumption in systems for catching harmful and toxic substances, it is necessary to provide a reduction of hydraulic resistance while maintaining the high efficiency of gas streams cleaning. Given the controversial requirements for equipment and despite the large number of existing machines for mass transfer processes, the development of new highly intensive and efficient equipment is of considerable interest to environmental protection technologies in many industries.

\section{Description of the problem}

The most common method of purifying gas streams is methods for the absorption of harmful components from the released industrial gases. In this case, either the process of physical absorption occurs, or the absorbent enters a chemical interaction with the absorbed component (the process of chemisorption).

Recently, the direction associated with conducting diffuse processes in intensive regimes with developed turbulence at high speeds of gas and liquid flows has become relevant. Actually turbolization of the gas-liquid system leads to an increase in the intensity of mass-exchange devices. One of the methods of turbulization of gas-liquid systems is their transformation into mobile unstable foam due to the kinetic energy of gas.

Foam mode and foam devices of the "classical" type are described in and analyzed in a lot of works. Intensified apparatuses with foam layer stabilizer have been widely used for capturing dust from gases and for gas absorption in the chemical and related industries. Due to its high efficiency, high unit capacity, good operational qualities of their application, they can improve the stages of gas purification for technological and sanitary purposes, increase the efficiency of mass transfer and the reliability of gas-cleaning equipment. Industrial introducing the stabilization method of the gas-liquid layer significantly expands the scope of foaming devices and opens up new possibilities for intensifying technological processes with the simultaneous creation of low-waste technologies.

Based on researching the semi-industrial models of foam machines with lattices, assembled from tubes of different diameters, a pilot sample of foam absorber with the productivity of $12000 \mathrm{~m}^{3} / \mathrm{h}$ of gas was designed [1].

One of the research directions was the absorption of ammonia by water in intensive foam modes with a stabilized foam layer. Absorption of ammonia by water is a typical process in absorber research to detect their effectiveness. 
The process of absorption of ammonia in foam mode on counterproductive gratings of the usual type is quite fully studied at gas velocities up to $2,5 \mathrm{~m} / \mathrm{s}$. In order to compare the technological parameters of ammonia absorption in water under similar conditions, the work of the most common industrial counter processing grating with an area of free intersection of 0,18 $\mathrm{m}^{2} / \mathrm{m}^{2}$ and a diameter of holes of $5 \mathrm{~mm}$ was investigated.

The research of this grating was carried out both with the stabilizer, and without it. The research was due to the need to determine the degree of effect of the stabilization of the layer and comparison with the technological parameters of the newly developed design foam apparatus, as well as the establishment of the general nature of dependencies and kinetic parameters of the absorption process.

In order to verify the results obtained during bench tests in laboratory conditions, a cycle of pilot-industrial tests was conducted in which the main gas components were ammonia, fluoride compounds (mainly silicon tetra fluoride). In the system of purifying gases from the spray dryer, a reconstruction of the existing absorber was carried out (hollow scrubber with three tiers of nozzles). Instead of nozzles in the scrubber case, a contact step with coarse hole gratings (the holes are $0,045 \times 0,045 \mathrm{~m}$ and $\mathrm{S}_{0}=0,28 \mathrm{~m}^{2} / \mathrm{m}^{2}$ ) and a foam layer stabilizer were installed.

\section{Conclusion}

The conducted studies indicate the possibility of deep cleaning and virtually complete removal of harmful components from gases in their separate absorption. Dependences of $\mathrm{NH}_{3}$ final content and fluorine in the gas after treatment from ammonium phosphate acidity shown that it is evident that the optimal mode for the absorption of these components lies within the $\mathrm{pH}$ of the solution from 3 to 5 . In this case, the maximum permissible emission rates for ammonia are maintained. This is confirmed by the high mass exchange characteristics of the developed foam device, allowing the process of joint and effective cleaning of these components in the range of $\mathrm{pH}$ irrigating solution from 3 to 5 [2].

\section{Acknowledgments}

As a result of experimental and industrial tests, it is established the following: the efficiency of dust capture practically does not depend on the $\mathrm{pH}$ of the solution and also on the increase of the inlet dust to $4-6 \mathrm{~g} / \mathrm{m}^{3}$. Increasing the density of the circulating pulp of ammonium phosphates does not affect the efficiency.

The conducted studies have experimentally confirmed the possibility of effective mass exchange processes in an intensive foam layer on counter currently coarse hinged gratings.

The application of new apparatus allows radically reconstructing, with minimal cost, technological schemes for gas cleaning in the chemical and other industries to provide them with better operational and technological characteristics, while simultaneously increasing the efficiency of working with concentrated solutions, their circulation and use in the main process.

\section{References}

[1] O. Liaposhchenko, O. Khukhryanskiy, V. Moiseev, M. Ochowiak, E. Manoylo. Intersification of foam layered apparatus by foam stabilization. Journal of Engineering Sciences, Vol. 5, Issue 2, pp. F13-F18, 2018, https://doi.org/10.21272/jes.2018.5(2).f3

[2] V.F. Moiseev, E. V. Manoylo, K. Yu. Repko, D. V. Davydov. Treatment of gas-discharge systems on tubular lattices with foam layer stabilizer. Bulletin of NTU "KhPI", № 53, pp. 114123, 2017, https://doi.org/10.20998/2413-4295.2017.53.17 